\title{
Optical Characterization of the Keck Array Polarimeter at the South Pole
}

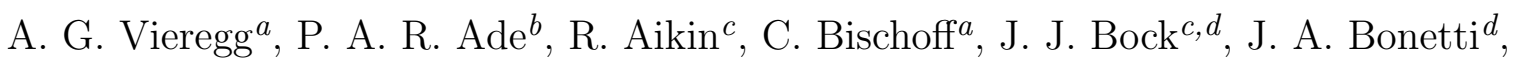
K. J. Bradford ${ }^{a}$, J. A. Brevik ${ }^{c}$, C. D. Dowell ${ }^{c, d}$, L. Duband ${ }^{e}$, J. P. Filippini ${ }^{c}$, S. Fliescher ${ }^{f}$,

S. R. Golwala ${ }^{c}$, M. S. Gordon ${ }^{a}$, M. Halpern ${ }^{g}$, G. Hilton ${ }^{h}$, V. V. Hristov ${ }^{c}$, K. Irwin ${ }^{h}$,

S. Kernasovskiy ${ }^{i}$, J. M. Kovac ${ }^{a}$, C. L. Kuo ${ }^{i}$, E. Leitch $^{j}$, M. Lueker $^{c}$, T. Montroy $^{k}$,

C. B. Netterfield ${ }^{l}$, H. T. Nguyen ${ }^{c, d}$, R. O’Brient ${ }^{c, d}$, R. W. Ogburn IV ${ }^{i}$, C. Pryke ${ }^{f}$, J. E. Ruhl ${ }^{k}$, M. Runyan ${ }^{c}$, R. Schwarz ${ }^{f}$, C. Sheehy ${ }^{f, j}$, Z. Staniszewskic ${ }^{c, d}$, R. Sudiwala ${ }^{b}$, G. Teply $^{c}$, J. Tolan $^{i}$, A. D. Turner ${ }^{d}$, P. Wilson ${ }^{d}$, and C. L. Wong ${ }^{a}$

${ }^{a}$ Harvard-Smithsonian Center for Astrophysics, 60 Garden Street, Cambridge, MA 02138, USA;

${ }^{b}$ Dept. of Physics and Astronomy, University of Wales, Cardiff, CF24 3YB, Wales, UK;

${ }^{c}$ California Institute of Technology, 1200 E. California Blvd., Pasadena, CA 91125, USA;

${ }^{d}$ Jet Propulsion Laboratory, 4800 Oak Grove Dr., Pasadena, CA 91109, USA;

eService des Basses Tempratures, DRFMC, CEA-Grenoble, 17 rue des Martyrs, 38054 Grenoble Cedex 9, France;

${ }^{f}$ School of Physics \& Astronomy, University of Minnesota, 116 Church Street S.E., Minneapolis, MN 55455, USA;

${ }^{g}$ Department of Physics \& Astronomy, University of British Columbia, 6224 Agricultural Road, Vancouver, BC V6T1Z1, Canada;

${ }^{h}$ NIST Quantum Devices Group, 325 Broadway, Boulder, CO 80305, USA;

${ }^{i}$ Stanford University, 382 Via Pueblo Mall, Stanford, CA 94305, USA;

jUniversity of Chicago, KICP, 933 E. 56th St., Chicago, IL 60637, USA;

${ }^{k}$ Physics Department, Case Western Reserve University, Cleveland, OH 44106, USA;

${ }^{l}$ Department of Physics, University of Toronto, Toronto, ON M5S 1A7, Canada;

\begin{abstract}
The Keck Array (SPUD) is a set of microwave polarimeters that observes from the South Pole at degree angular scales in search of a signature of Inflation imprinted as B-mode polarization in the Cosmic Microwave Background (CMB). The first three Keck Array receivers were deployed during the 2010-2011 Austral summer, followed by two new receivers in the 2011-2012 summer season, completing the full five-receiver array. All five receivers are currently observing at $150 \mathrm{GHz}$. The Keck Array employs the field-proven BicEP/BiceP2 strategy of using small, cold, on-axis refractive optics, providing excellent control of systematics while maintaining a large field of view. This design allows for full characterization of far-field optical performance using microwave sources on the ground. We describe our efforts to characterize the main beam shape and beam shape mismatch between co-located orthogonally-polarized detector pairs, and discuss the implications of measured differential beam parameters on temperature to polarization leakage in CMB analysis.
\end{abstract}

Keywords: Cosmic Microwave Background, polarization, Inflation, The Keck Array

Send correspondence to A. G. Vieregg, 60 Garden Street, MS 42, Cambridge, MA 02138 USA, E-mail: avieregg@cfa.harvard.edu

Millimeter, Submillimeter, and Far-Infrared Detectors and Instrumentation for Astronomy VI, edited by Wayne S. Holland, Jonas Zmuidzinas, Proc. of SPIE Vol. 8452,

845226 (C) 2012 SPIE - CCC code: 0277-786X/12/\$18 - doi: 10.1117/12.926639

Proc. of SPIE Vol. 8452 845226-1 


\section{INTRODUCTION}

Cosmological Inflation is a theory that describes the entire observable Universe as a microscopic volume that underwent violent, exponential expansion during the first fraction of a second. Inflation is supported by the flatness and extreme uniformity of the Universe observed through measurements of the Cosmic Microwave Background (CMB). ${ }^{1,2}$ Measurements of the polarization of the CMB could prove to be an impressive tool for probing the epoch of Inflation. A generic prediction of Inflation is the production of a Cosmic Gravitational-Wave Background, which in turn would imprint a faint but unique signature in the polarization of the CMB that has a curl component. ${ }^{3,4}$ This curl component of the polarization field is commonly called B-mode polarization, while the curl-free component, dominated by production due to density fluctuations at the time of last scattering, is called E-mode polarization. The strength of the B-mode polarization signature depends on the energy scale of Inflation, and would be detectable if Inflation occurred near the energy scale at which the fundamental forces unify $\left(\sim 10^{16} \mathrm{GeV}\right)$.

The Keck Array, also called SPud, is a set of five degree-scale microwave polarimeters that is currently observing the CMB from the Martin A. Pomerantz Observatory at the South Pole in search of a B-mode polarization signature from Inflation. ${ }^{5}$ Each of the five receivers has 512 Transition Edge Sensor (TES) detectors, 16 of which are dark, leaving 496 detectors that are coupled to planar arrays of slot antennas, for a total of 2480 optical detectors in the entire instrument. Receivers utilize a compact, on-axis refracting telescope design.

The first three Keck Array receivers were deployed during the 2010-2011 Austral summer and two new receivers followed in the 2011-2012 deployment season. The first season of observation with the full five-receiver array is currently underway, with all receivers observing at $150 \mathrm{GHz}$. The modular design of the Keck Array allows for future upgrades to include replacement of individual receivers to provide additional frequency coverage at $100 \mathrm{GHz}$ and $220 \mathrm{GHz}$. The Keck Array leverages field-proven techniques employed for the BicEP and BICEP2 telescopes, but with a vastly increased number of detectors, leading to increased sensitivity to the tiny Inflationary B-mode signal. The current upper limit on the B-mode amplitude in the CMB is set by the Keck Array's predecessor experiment, Bicep, ${ }^{6,7}$ and corresponds to $r<0.72$ at $95 \%$ confidence level, where $r$ is the tensor-to-scalar ratio. The Keck Array aims to reach a sensitivity corresponding to $r=0.01$, where gravitational lensing of E-modes into B-modes should begin to be comparable in strength to the Inflationary signal.

As sensitivity dramatically improves with each generation of experiments, control of systematics becomes increasingly important. Precise characterization of the optical performance of Keck Array receivers is critical to reach these ambitious sensitivity goals. In the simplest mapmaking schemes, differential beam effects between co-located orthogonally-polarized pairs of detectors can lead to leakage of the CMB temperature signal into the much smaller B-mode signal, potentially limiting the ability of the Keck Array to reach its design sensitivity if these systematics are not well understood.

Characterizing the beam pattern of each of the 2480 Keck Array detectors in the far field presents a challenge in both data acquisition and reduction. We describe here our effort to characterize the Keck Array optical performance through an extensive ground-based precision beam mapping campaign at the South Pole, and discuss our understanding of the cause of measured beam non-idealities and our strategy for mitigating the effect of measured differential beam components in CMB analysis.

Four companion papers are also presented at this conference, focusing on the status of BICEP2 and the Keck Array (Ogburn et al..$^{8}$ ), the sensitivity of the Keck Array (Kernasovskiy et al. ${ }^{9}$ ), the performance of the dualpolarization planar antenna array (O'Brient et al. ${ }^{10}$ ), and the thermal stability of BiCEP2 (Kaufman et al. ${ }^{11}$ ).

\section{OPTICAL DESIGN}

Each Keck Array receiver is a compact, single-frequency, on-axis refractive telescope with an aperture of $26.4 \mathrm{~cm}$, designed to maintain tight control of systematics. All optical elements are cold $(4 \mathrm{~K}$ or $50 \mathrm{~K})$ to maintain low and stable optical loading on the focal plane. Figure 1 shows a schematic of the BICEP2/Keck Array optical chain. An in-depth discussion of the design of BICEP2 optical elements can be found in Aikin et al. ${ }^{12}$

The eyepiece and objective lenses are made of high-density polyethylene (HDPE) and are designed to provide even illumination of the aperture, which is coincident with the objective lens. The illumination at the edge of 


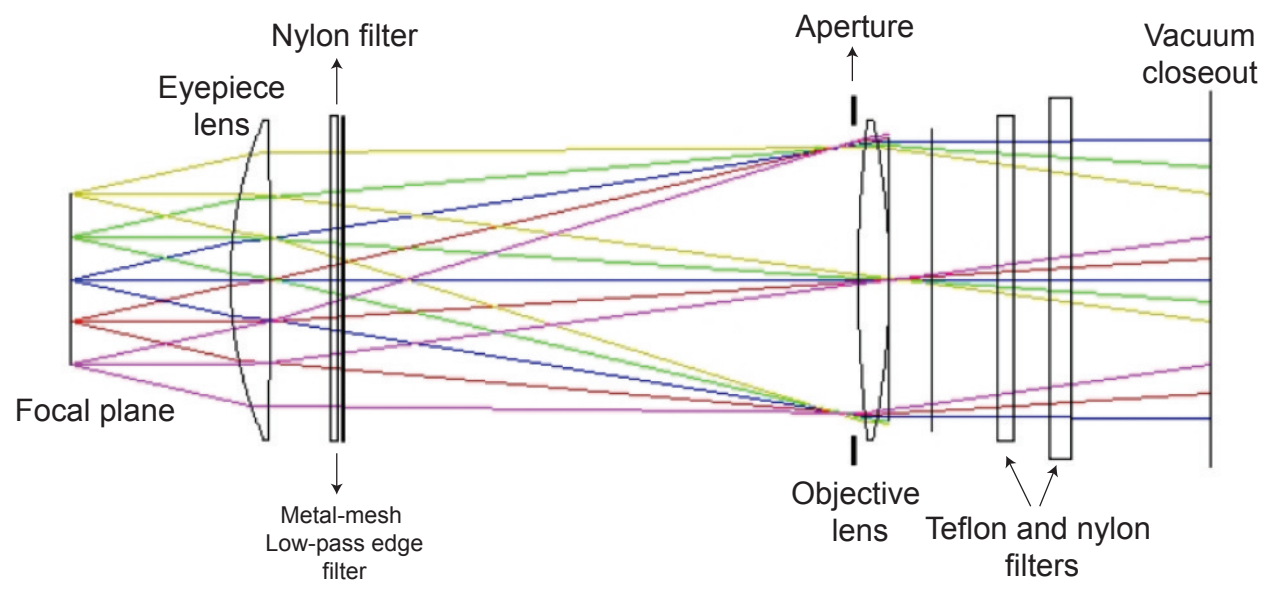

Figure 1: A schematic of the BICEP2/Keck Array optical chain, from Aikin et al. ${ }^{12}$

the aperture compared to the illumination at the center of the aperture is designed to be $-12.4 \mathrm{~dB}$ for detectors at the center of the focal plane. Both lenses as well as the telescope housing and aperture are cooled to $4 \mathrm{~K}$. Two Teflon, two nylon, and one metal-mesh filter block IR radiation from reaching the focal plane. The two Teflon filters and one nylon filter are located in front of the objective lens, and are held at $50 \mathrm{~K}$. Another nylon filter and the metal-mesh filter sit at $4 \mathrm{~K}$ between the objective and eyepiece lenses inside the telescope itself to further reduce loading. Teflon has excellent in-band transmission at cryogenic temperatures. While nylon has higher in-band transmission loss, it has a steeper transmission rolloff, providing significant reduction of far-IR loading on the sub-Kelvin stages. The metal-mesh filter is a low pass filter with a cutoff at $250 \mathrm{GHz}$, providing additional blocking of out-of-band power.

Keck Array optical elements are anti-reflection coated with porous Teflon with an index of refraction matched to the optical element and thickness matched to the observing frequency of each receiver, currently $150 \mathrm{GHz}$. Future upgrades to the array include receivers that observe at 100 and $220 \mathrm{GHz}$, which require different antireflection coatings but contain an otherwise identical optical system.

The vacuum window has a $32 \mathrm{~cm}$ clear aperture, making design and construction of strong and durable vacuum windows a challenge. Keck Array vacuum windows are made of Zotefoam HD30, a nitrogen-expanded polyethylene foam that was chosen for its high microwave transmission, its strength against deflection under vacuum, and its adhesion strength to epoxy used to bond the foam to an aluminum frame.

To reduce sidelobe pickup, individual co-moving ground shields are installed in front of each receiver's vacuum window. These forebaffles are coated on the inside with HR10 microwave absorber and a weatherproofing foam, providing good termination of sidelobes. The forebaffles intersect radiation at $9.5^{\circ}$ off of the boresight axis from the edge of the vacuum window.

The BICEP2 and Keck Array optical designs are identical except for a few small differences. The material used for the vacuum window for BICEP2 is Zotefoam PPA30, but is Zotefoam HD30 for the Keck Array. BICEP2 has a larger forebaffle than Keck Array receivers. The exact configuration of the IR blocking filters is different between the two experiments; BICEP2 has one Teflon filter at $40 \mathrm{~K}$, another at $100 \mathrm{~K}$, and only has one nylon filter (at $4 \mathrm{~K})$.

\section{OPTICAL CHARACTERIZATION}

\subsection{Near-Field Beam Characterization}

To characterize aperture illumination, we measure the near-field beam pattern of each Keck Array detector using a chopped thermal source mounted on an x-y translation stage attached to the cryostat above the vacuum window, as close to the aperture stop of the telescope as possible. In practice, the source is about $30 \mathrm{~cm}$ above 
the aperture. Figure 2 shows the beam pattern of two example detectors in the near field. The left panel shows the beam pattern of a detector near the center of the focal plane, which evenly illuminates the aperture. The right panel shows the beam pattern of a detector near the edge of the focal plane that is significantly truncated by the aperture because of non-ideal beam pointing at the focal plane (worst case). This type of truncation translates to some ellipticity in the beam pattern in the far field and only affects a small fraction of detectors.
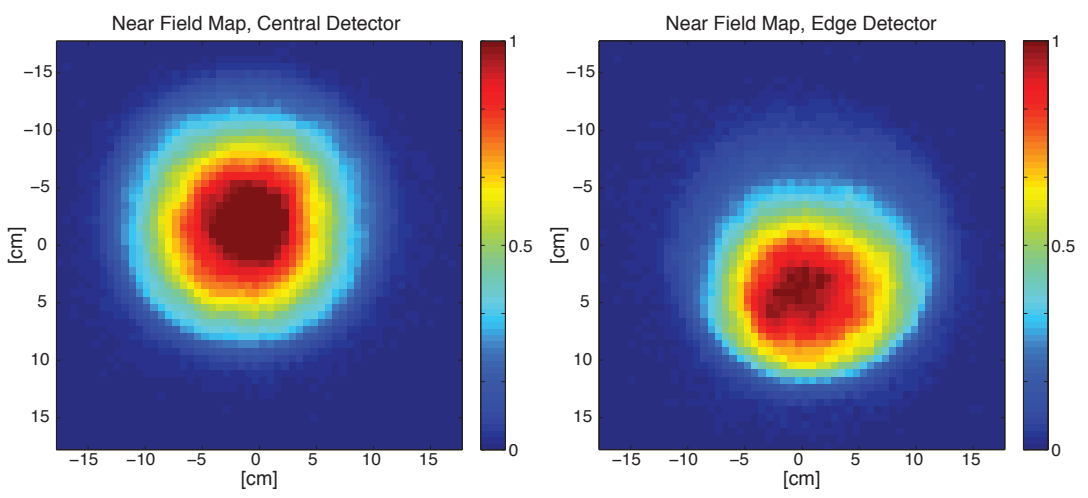

Figure 2: Measurement of the near-field beam pattern for two example Keck Array channels, measured above the aperture stop at the vacuum window. Left: A detector near the center of the focal plane; Right: A detector near the edge of the focal plane, showing significant truncation by the aperture (worst case).

\subsection{Polarization Angle and Polarization Efficiency Characterization}

We must know the polarization angle, $\psi$, and cross-polar response, $\epsilon$, of each detector to correctly construct polarization maps of the sky. The polarization efficiency is calculated from the cross-polar response as $(1-\epsilon) /(1+\epsilon)$. BICEP and BICEP2 measured these polarization parameters using a polarized broadband amplified microwave source by rotating the telescope about its boresight while the source polarization remained fixed. ${ }^{7}$ For BICEP2, the polarization efficiency was measured to be $<1 \%$, with leakage dominated by inductive crosstalk between front-end SQUIDs in the readout system. ${ }^{12}$ This technique, however, would be tedious with the Keck Array because as we rotate the drum that houses the receivers to rotate the receivers about their boresights, the physical location of each receiver also moves, making the data set much more difficult to take and analyze than it was for BICEP and BICEP2.

To measure the polarization angle and cross-polar response of each detector without having to rotate the drum, we have developed a new rotating polarized broadband amplified microwave source (see Figure 3). The source emits radiation in the 140-160 GHz range, designed to cover the passband of the current Keck Array receivers. A $50 \Omega$ load provides room-temperature thermal noise at the input of the first stage of amplification (80 dB). A series of frequency multipliers, amplifiers, and filters bring the output frequency to the desired range $(140-160 \mathrm{GHz})$. Linearly polarized radiation is emitted by a $15 \mathrm{~dB}$ gain horn antenna, and is further polarized by a free-standing wire grid, yielding cross-polar leakage of the source $<0.03 \%$. Two variable attenuators in series allow for control of output power over a large dynamic range, making the source useful for far-field beam mapping as well as sidelobe mapping with the source closer to the receiver. A microwave switch chops the source at $\sim 10 \mathrm{~Hz}$. The entire source is mounted on a stepped rotating stage and has a total positional repeatability $<0.01^{\circ}$.

We used the rotating polarized source to repeat a measurement of detector polarization angles and cross-polar response for BICEP2 in February 2012. An example of the polarization modulation vs. source angle for one pair of BICEP2 detectors is shown in Figure 3. The orthogonally-polarized detectors in the pair are $90^{\circ}$ out of phase. We plan to make similar measurements with the Keck Array during the upcoming 2012-2013 summer season. 

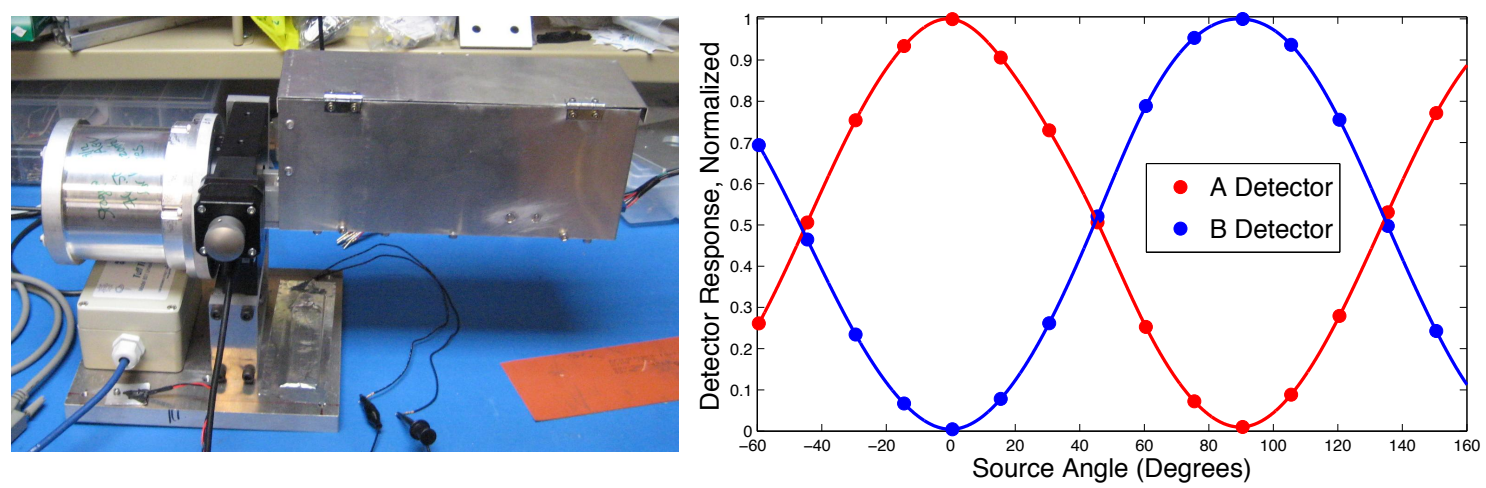

Figure 3: Left: The rotating polarized amplified thermal broadband noise source used for polarization characterization. Right: Polarization modulation vs. source angle of an example detector pair from BICEP2, measured using the rotating polarized source.

\subsection{Far-Field Beam Characterization}

\subsubsection{Data Acquisition and Reduction}

Measuring the beam pattern of each of the 2480 detectors of the Keck Array in the far field presents a challenge and requires an extensive beam mapping campaign at the South Pole. Figure 4 shows the setup used for measuring the beam pattern in the far field. With all five receivers installed in the drum, we install a $1.2 \times 1.8 \mathrm{~m}$ aluminum honeycomb mirror, flat to $0.2 \mathrm{~mm}$ across the mirror, mounted on carbon-fiber rods. The mirror assembly was designed to be installed with no overhead crane; the entire system weighs less than $150 \mathrm{~kg}$. The mirror redirects the beams over the top of the ground shield and to a chopped thermal microwave source with an aperture of $20 \mathrm{~cm}$ mounted on a $10 \mathrm{~m}$ tall mast on the Dark Sector Laboratory, $211 \mathrm{~m}$ away. The thermal source chops between a flat mirror directed to zenith $(\sim 15 \mathrm{~K})$ and ambient $(\sim 260 \mathrm{~K})$ at a tunable frequency, set to be $10 \mathrm{~Hz}$.
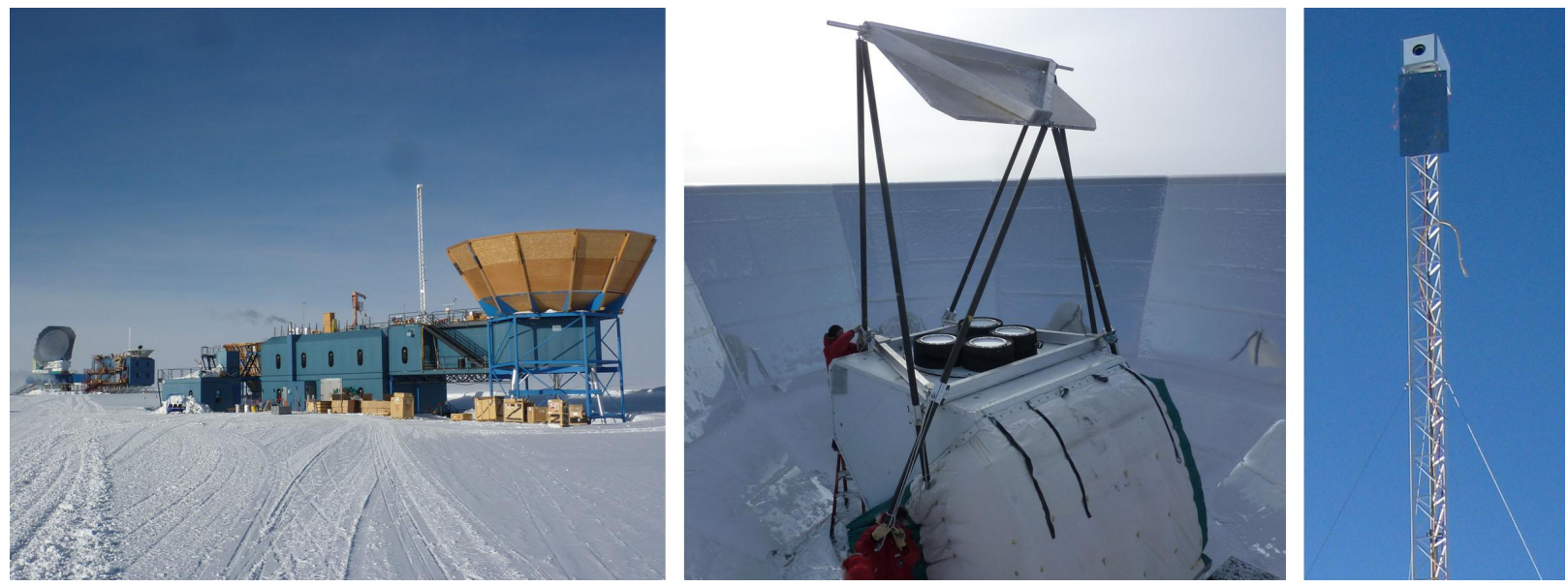

Figure 4: The setup for measuring far-field beam pattern of Keck Array detectors in situ at the South Pole. A chopped, broadband, microwave source broadcasts from a mast on the Dark Sector Laboratory (DSL), and a large aluminum honeycomb mirror is installed to redirect the beams of the Keck Array to the source. Left: The Keck Array in the foreground, with DSL in the background. Middle: The aluminum honeycomb mirror, installed on the Keck Array for beam measurements. Right: The microwave source mounted on a mast on the roof of DSL. 
The size of the mirror allows us to map only three receivers at a time. By rotating the drum below the mirror, we can move selected receivers into a position under the mirror for beam mapping. For each receiver, we take data at five different drum angles so that we can check that a rotation of a receiver underneath the mirror does not affect the measurement. The five drum rotations used for each receiver are separated by $36^{\circ}$, for a total drum rotation coverage of $144^{\circ}$ for each receiver, the maximum we could achieve using any single mirror position.

The data set discussed here consists of five measurements (one at each of five drum rotations) of each of the five Keck Array receivers, each with 496 optically-coupled detectors. This data set was taken in February 2012, at the end of the most recent summer season. We filter the chop reference signal to match the filtering that occurs in the readout system and then demodulate the timestream data. The reflection off the mirror and parallax effects are handled with a pointing model that describes the Keck Array mount system as well as the mirror used for beam mapping.

\subsubsection{Beam Parameters}

Figure 5 displays measured beam maps for all detectors of a single polarization (denoted "A" polarization) over the entire array. We fit an elliptical Gaussian to the main beam for each detector, according to

$$
g e^{-\frac{1}{2}(\vec{x}-\vec{r}) \Sigma^{-1}(\vec{x}-\vec{r})}
$$

where $\vec{r}$ is the location of the beam center, $g$ is the amplitude, and $\Sigma$ is the covariance matrix. Ellipticity parameters, $p$ and $c$, corresponding to amplitudes of the the "plus" and "cross" ellipticity orientations, are defined below in Equation 2 as part of the covariance matrix.

$$
\Sigma=\left[\begin{array}{cc}
\sigma^{2}(1+p) & c \sigma^{2} \\
c \sigma^{2} & \sigma^{2}(1-p)
\end{array}\right]
$$

Figure 6 shows an example map, the elliptical Gaussian fit, and the fractional residual after subtracting the fit. Average beam widths and ellipticities are shown in Table 1 for each receiver. The average beam width $(\sigma)$ over the array is $0.215 \pm 0.007^{\circ}$. Figure 7 shows the measured far-field beam profile, averaged over all detectors in one Keck Array receiver.

\begin{tabular}{|l|c|c|c|c|c|}
\hline \multirow{2}{*}{ Parameter } & \multicolumn{5}{|c|}{ Keck Array 2012 Receiver-Averaged Beam Parameter Values } \\
& Receiver 0 & Receiver 1 & Receiver 2 & Receiver 3 & Receiver 4 \\
\hline \hline Beam width $(\sigma$, degrees $)$ & $0.214 \pm 0.005$ & $0.213 \pm 0.006$ & $0.213 \pm 0.006$ & $0.216 \pm 0.008$ & $0.218 \pm 0.013$ \\
Beam ellipticity ${ }^{1}$ & $0.010 \pm 0.007$ & $0.012 \pm 0.006$ & $0.012 \pm 0.007$ & $0.013 \pm 0.010$ & $0.013 \pm 0.010$ \\
\hline
\end{tabular}

Table 1: Receiver-averaged single beam parameters for all five Keck Array receivers with the standard deviation of these parameters across each receiver. The spread is dominated by real detector-to-detector scatter, not measurement uncertainty for individual detectors.

\subsubsection{Differential Beam Parameters}

The beam pattern of a single detector can also be characterized as a set of perturbations on an idealized circular Gaussian fit, with a nominal width $\left(\sigma_{\mathrm{n}}\right)$ equal to the receiver-averaged value and a nominal beam center equal to the calculated center for the pair of detectors from the initial elliptical Gaussian fit. We consider the first six perturbations, corresponding to the templates shown in Figure 8. These six templates correspond to relative responsivity, x-position offset, y-position offset, beam width, ellipticity in the "plus" orientation, and ellipticity in the "cross" orientation.

We calculate the regression coefficient for each measured individual beam pattern against each template map. Differential beam parameters for a pair of co-located orthogonally-polarized detectors, which we denote "A" and "B" detectors, are then the difference of the regression coefficients for each detector. Table 2 gives the 


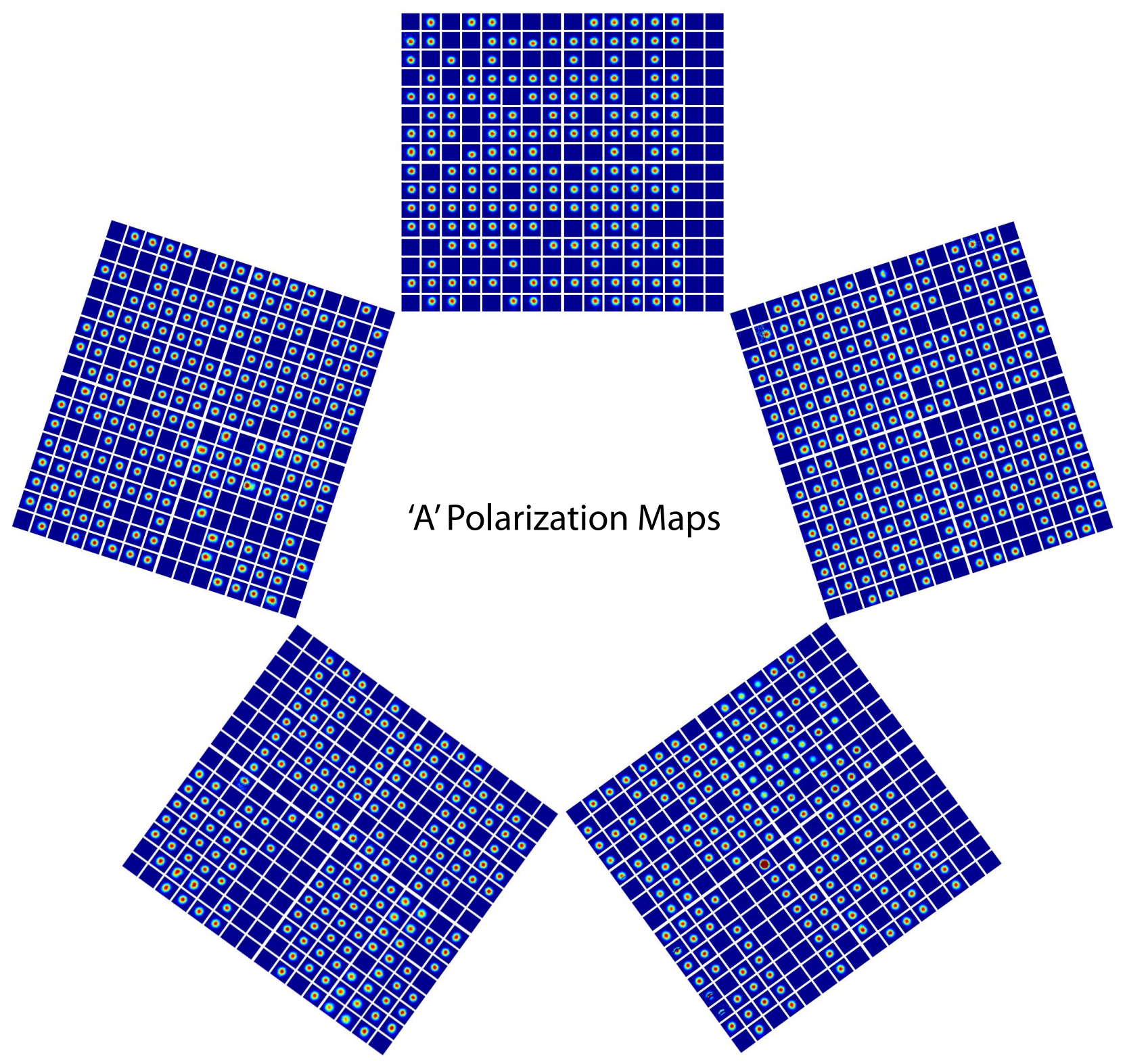

Figure 5: Measured beam maps of A polarization detectors for the entire array. The maps are arranged in a layout that represents the location and orientation of the five receivers in the drum, and does not represent the field of view. In the far field, the five receivers have completely overlapping fields of view, and the beam centers are more closely spaced than shown here.

correspondence between these differential beam parameters and the associated elliptical Gaussian fit parameters for each beam map (Equations 1 and 2) in the limit of small perturbations.

In this analysis, we extract differential beam parameters by calculating regression coefficients of individual detector beam maps against each of the template maps and then differencing regression coefficients for each detector pair. This proves convenient when predicting and mitigating beam mismatch-induced polarization, as the leakage scales linearly with these differential parameters. A full description of this parameterization and its implementation in analysis can be found in Aikin et al. ${ }^{13}$ 

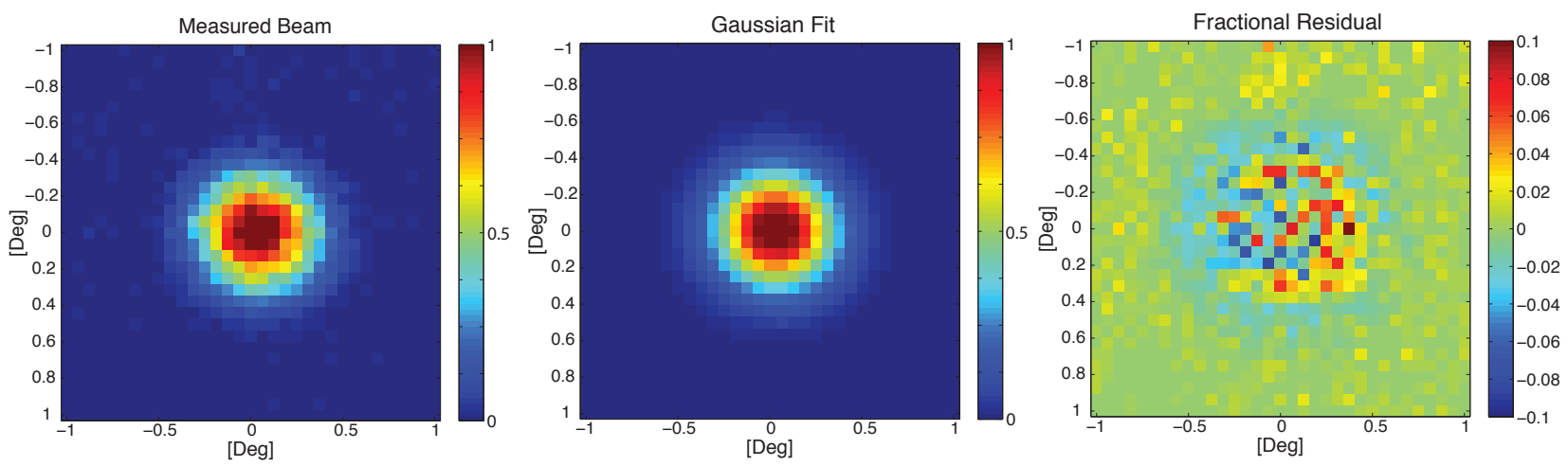

Figure 6: Left: Example measured far-field beam pattern, linear scale. Middle: Gaussian fit to measured beam pattern. Right: Fractional residual after subtracting the Gaussian fit in the middle panel. Note: Right-hand panel has a different color scale than the left two panels.

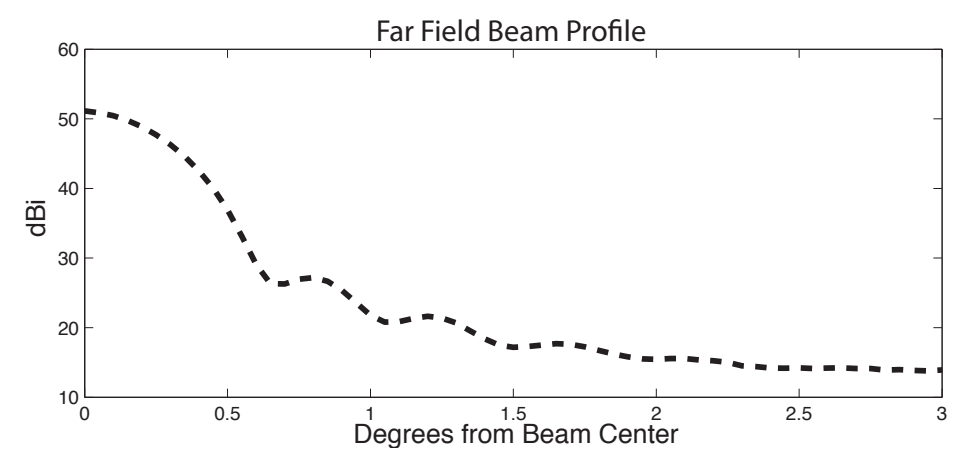

Figure 7: Radial profile of the Keck Array far-field beam pattern, averaged over one receiver. The profile shows the first few sidelobes and nulls before the beam pattern falls to the noise floor of the measurement.

We can calculate the regression coefficient for five of the templates shown in Figure 8 from beam maps, and extract differential beam parameters for each detector pair. The sixth coefficient, for the relative responsivity, is equivalent to differential gain which is calibrated frequently during CMB observations.

Figure 9 shows an example of this decomposition for a representative detector pair. The first column shows the normalized A beam map, the second column shows the normalized B beam map, and the third column shows the difference between the normalized A and B maps. We construct a model, shown in the second row, by summing the position offset, beam width, and ellipticity components calculated via template regression for each detector. The residual after subtracting the model is shown in the third row. The fourth column explicitly shows the differential components (templates scaled by the regression coefficient) that are summed to give the model for the normalized A-B difference map. The normalization explicitly sets the responsivity mismatch to zero.

Histograms of all differential parameters for each detector pair in the Keck Array are shown in Figure 10 and receiver-averaged values are shown in Table 3. Each measurement of each detector's main beam must pass a set of criteria to be included in the final extraction of beam parameters, including a check that the beam center was not near the edge of the mirror and excluding measurements where the initial elliptical Gaussian fit failed. Note that the relative amplitudes of the distinct differential parameters shown in the histograms and in Table 3 do not reflect the relative map amplitudes (templates scaled by the regression coefficient).

This set of measurements of single normalized A-B difference residuals is noise-dominated (see Figure 9). Increased signal-to-noise beam map data would help improve the data quality. We will have the opportunity 


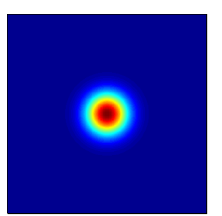

(a)

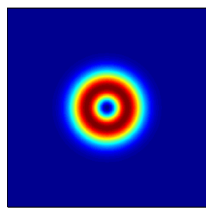

(d)

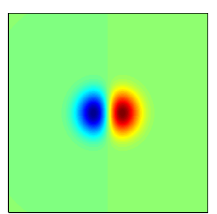

(b)

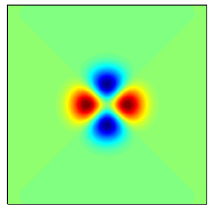

(e)

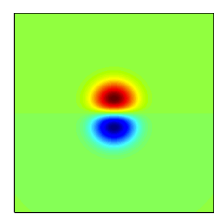

(c)

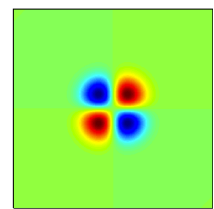

(f)

Figure 8: Differential beam templates resulting in mismatch in (a) responsivity (b) x-position (c) y-position (d) beam width (e) ellipticity in plus (f) ellipticity in cross. In the limit of small differential parameters, a differenced beam pattern constructed from the difference of two elliptical Gaussians can be represented as a linear combination of each of these templates. From Aikin et al. ${ }^{13}$

\begin{tabular}{|l|c|}
\hline Parameter & Definition \\
\hline \hline Differential relative responsivity $(\delta g)$ & $\Delta\left(g_{A} / g_{\mathrm{n}}\right) /\left(g_{B} / g_{\mathrm{n}}\right)$ \\
Differential pointing in $x\left(\delta r_{x}\right)$ & $\left(\vec{r}_{a}-\vec{r}_{b}\right) \cdot \hat{x} / 2 \sigma_{\mathrm{n}}$ \\
Differential pointing in $y\left(\delta r_{y}\right)$ & $\left(\vec{r}_{a}-\vec{r}_{b}\right) \cdot \hat{y} / 2 \sigma_{\mathrm{n}}$ \\
Differential beam width $(\delta \sigma)$ & $\left(\sigma_{A}^{2}-\sigma_{B}^{2}\right) / \sigma_{\mathrm{n}}^{2}$ \\
Differential ellipticity, plus orientation $(\delta p)$ & $\left(p_{A}-p_{B}\right) / 2$ \\
Differential ellipticity, cross orientation $(\delta c)$ & $\left(c_{A}-c_{B}\right) / 2$ \\
\hline
\end{tabular}

Table 2: The correspondence between differential beam parameters and the associated elliptical Gaussian fit parameters for each beam map in the limit of small perturbations. The two unitless parameters corresponding to differential ellipticity, $p$ and $c$, are the ellipticity parameters defined in Equation 2. $\sigma_{\mathrm{n}}$ is the receiver-averaged beam width, $\vec{r}_{a}$ and $\vec{r}_{b}$ are the vector from the averaged detector-pair center to the $\mathrm{A}$ or B beam center, $\sigma_{A}$ and $\sigma_{B}$ are the beam width for $\mathrm{A}$ and $\mathrm{B}, g_{A}$ and $g_{B}$ are the responsivity for $\mathrm{A}$ and $\mathrm{B}$, and $g_{\mathrm{n}}$ is the nominal receiver-averaged responsivity. This table is from Aikin et al. ${ }^{13}$

to repeat this beam mapping campaign during the upcoming 2012-2013 deployment season, but with a higher signal level chopped thermal source and an amplified circularly polarized broadband noise source.

\section{MODELED DIFFERENTIAL BEAM EFFECTS}

The largest of the clearly modeled differential beam effects is the pointing mismatch, indicating that the Keck Array has a pointing offset between orthogonally-polarized detector pairs that averages to $1.6-3.1 \%$ of the beam full width at half maximum (FWHM) for different receivers. We have spent a great deal of effort characterizing the mismatch and investigating its possible sources. A similar level of mismatch is observed in BICEP2, ${ }^{12}$ but was not present in BICEP, ${ }^{7}$ which used horn antennas rather than planar antenna arrays. BICEP had a very similar optical design to BICEP2 and the Keck Array, using the same materials for lenses and filters.

We have measured the pointing offset extensively in both the near field and the far field, in testbed locations in North America as well as in situ at the South Pole. We have seen some evidence that the amplitude of the far-field offset scales with the near-field offset. However, there appears to be a complex relationship between the overall observed near-field mismatch and the overall far-field mismatch.

Since deploying the Keck Array to the South Pole, we have made great progress in reducing the size of the near-field mismatch arising at the focal plane. This effort is described in detail in O'Brient et al. ${ }^{10}$ a companion paper at this conference. Recent measurements have revealed that newly-produced replacement Keck Array 

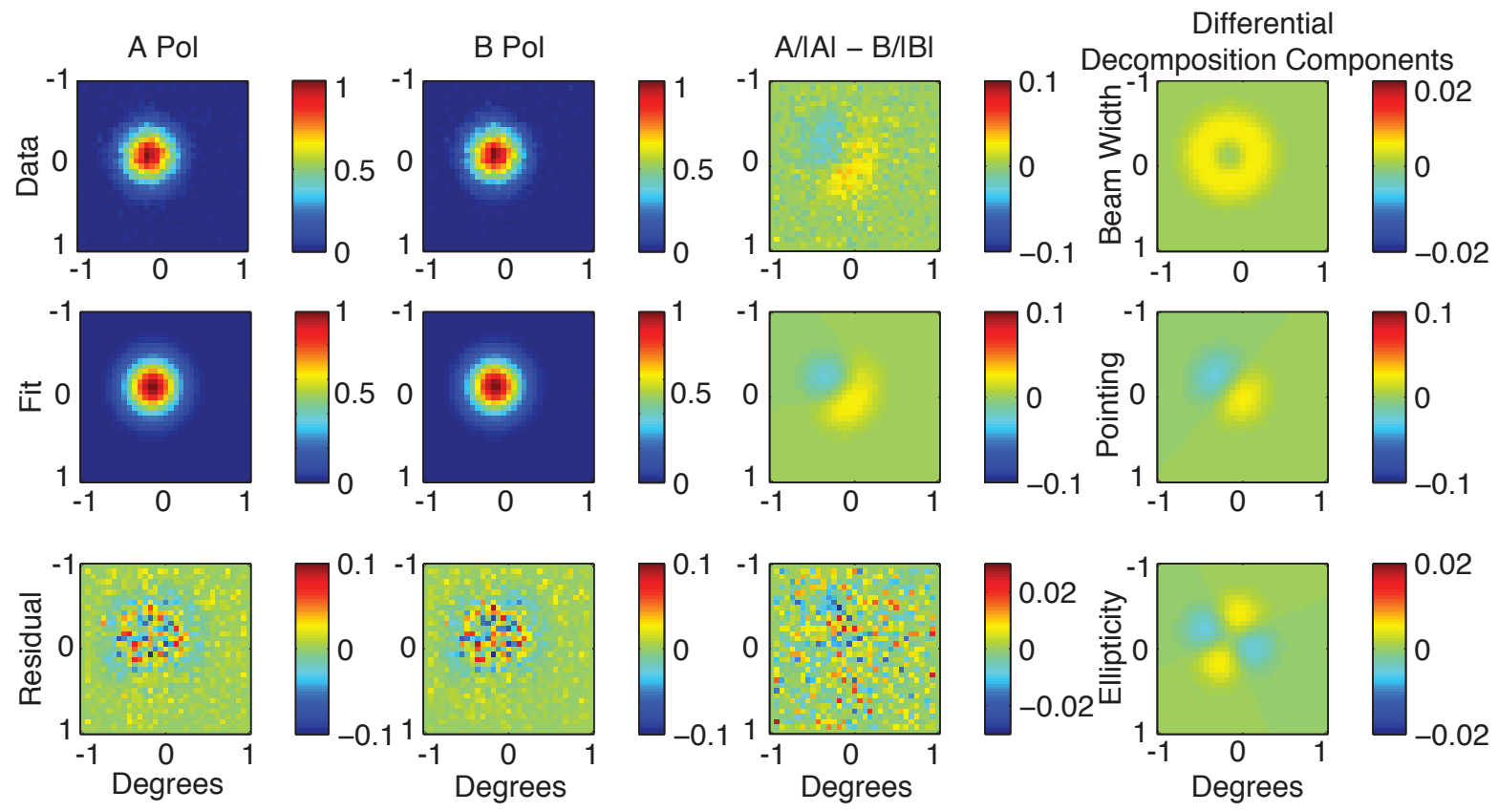

Figure 9: Example plot of the linear basis fit parameter calculation for one pair of detectors. The first column is the A beam pattern (normalized), the second column is the B beam pattern (normalized), the third column is A-B; for these three columns, the first row is the measured map, the second row is the fit, and the third row is the residual. The fourth column is the different decomposition components (the template multiplied by the regression coefficient).
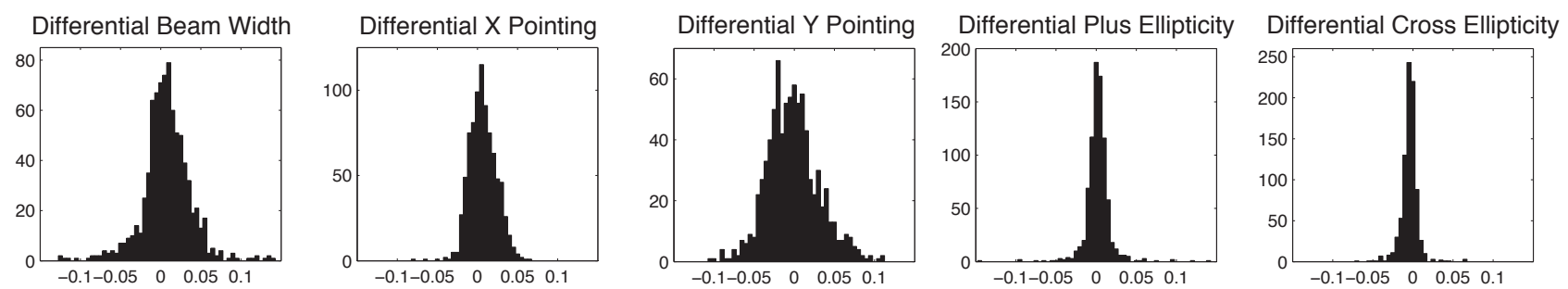

Figure 10: Histograms of differential beam parameters. Regression coefficients for each of the templates shown in Figure 8 are calculated individually for A and B detectors in a pair and are then differenced to extract differential beam parameters.

detector tiles exhibit a near-field mismatch that is a factor of 10 smaller than in focal planes currently installed at the South Pole.

Upcoming detailed measurements of the new reduced near-field mismatch focal planes in the far field will be very informative. We expect a reduction of the far-field mismatch, but because of the apparent complex relationship between the near and far-field effects, we do not necessarily expect a linear scaling between the measured reduction of the near-field mismatch and the far-field mismatch.

\section{RESIDUAL DIFFERENTIAL BEAM EFFECTS}

Temperature to polarization leakage in $\mathrm{CMB}$ maps that arises from the linearly-modeled components of the differential beams described in the previous section can be mitigated in analysis. A discussion of the methods we 


\begin{tabular}{|l|c|c|c|c|c|}
\hline Parameter & Receiver 0 & Receiver 1 & Receiver 2 & Receiver 3 & Receiver 4 \\
\hline \hline Differential beam width $(|\delta \sigma|)$ & $0.014 \pm 0.015$ & $0.024 \pm 0.024$ & $0.025 \pm 0.035$ & $0.023 \pm 0.020$ & $0.031 \pm 0.028$ \\
Differential pointing $\left(\sqrt{\delta r_{x}^{2}+\delta r_{y}^{2}}\right)$ & $0.033 \pm 0.018$ & $0.037 \pm 0.016$ & $0.019 \pm 0.014$ & $0.031 \pm 0.024$ & $0.035 \pm 0.024$ \\
Differential ellipticity $\left(\sqrt{\delta p^{2}+\delta c^{2}}\right)$ & $0.011 \pm 0.007$ & $0.013 \pm 0.008$ & $0.011 \pm 0.012$ & $0.013 \pm 0.011$ & $0.015 \pm 0.015$ \\
\hline
\end{tabular}

Table 3: Receiver-averaged differential beam parameters with the standard deviation of these parameters across each receiver. Regression coefficients for each of the templates shown in Figure 8 are calculated individually for $\mathrm{A}$ and $\mathrm{B}$ detectors in a pair and are then differenced to extract differential beam parameters. Both real scatter among detector pairs and measurement uncertainty for individual detector pairs contribute to the spread, but measurement uncertainty is subdominant, especially for the differential pointing parameter

are implementing to reject these linear components in CMB mapmaking, which does not rely on measurement of their amplitude, can be found in Aikin et al. ${ }^{13}$ After this mitigation, temperature to polarization leakage due to any unmodeled residual differential beam shape will remain.

Figure 11 shows power vs. angular scale for an example measured beam pattern and for the differential A-B residual after removing the extracted differential components. The A-B residual describes the higher-order differential beam effects that remain after mitigation of leading-order effects. To reduce the noise floor of the measurement of this residual, we have averaged over all five measurements of these detectors after accounting for drum orientation rotation. Still, because of the limited signal-to-noise of these measurements, the residual power shown here is only an upper limit on the actual residual differential power. We intend to improve the beam map measurements next season to achieve higher signal-to-noise measurements by using brighter sources.

At $\ell=100$, where our sensitivity to the Inflationary B-mode spectrum is best, the ratio between the unmodeled residual differential power that will remain after mitigation of leading-order effects in analysis and the power in the main beam is at most at the $10^{-5}$ level for one typical pair of detectors only. This upper limit is already close to the raw rejection ratio needed to probe polarization to a level of $r=0.01$. In actual CMB observation, we also benefit enormously from averaging-down effects from observing the sky with many detectors and at multiple drum angles. Full simulations of these effects are planned, and together with improved beam measurements to constrain residual differential power to lower levels, the situation looks promising for control of beam systematics to below the $r=0.01$ level.

\section{CONCLUSIONS}

Pushing deeper into the level of B-mode polarization to constrain $r$ requires the dramatically increased sensitivity that the Keck Array provides, but also requires tight control of systematics. Through a massive beam mapping campaign, we have measured beam properties of each of the 2480 detectors in the Keck Array. We extract differential beam parameters using the same linear basis that will be employed for analysis mitigation of these modeled effects. The source of the dominant pointing mismatch is still under investigation. We believe that there is a complex relationship between the size and orientation of the near-field mismatch and that of the far field.

Measurements with new reduced near-field mismatch focal planes in the far field are upcoming. We expect that the next generation of Keck Array focal planes will benefit from dramatically reduced far-field mismatch. For the current receivers, we have shown that noise-dominated upper limits placed on the unmodeled residual component of the difference beam pattern already reach the $10^{-5}$ level at $\ell=100$ when including one pair of detectors only. We are optimistic that improved constraints on this residual, together with full simulations which account for averaging-down effects from observing the sky with many detectors at multiple drum angles, will demonstrate beam systematic control more than sufficient to reach $r=0.01$.

\section{ACKNOWLEDGMENTS}

The Keck Array is supported by the National Science Foundation, Grant No. ANT-1044978/ANT-1110087, and by the Keck Foundation. AGV gratefully acknowledges support from the National Science Foundation, Grant No. ANT-1103553. We are also grateful to Robert Schwarz for spending the winter at the South Pole in 


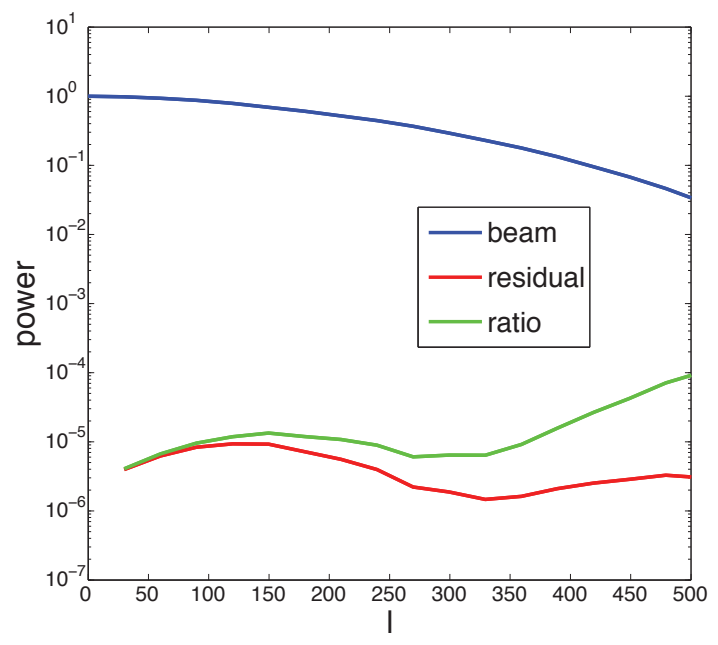

Figure 11: Power vs. angular scale for a typical example beam pattern (blue) and the residual beam component after removing leading-order differential components (red). The maps are shown in Figure 9. The ratio of the two is shown in green.

both 2011 and 2012 for this project, and to the South Pole Station logistics team. We thank our BicEP2, Keck Array, and SPIDER colleagues for useful discussions and shared expertise.

\section{REFERENCES}

[1] de Bernardis, P. et al., "A flat Universe from high-resolution maps of the cosmic microwave background radiation," Nature 404, 955-959 (Apr. 2000).

[2] Peiris, H. V. et al., "First-Year Wilkinson Microwave Anisotropy Probe (WMAP) Observations: Implications For Inflation," Astrophys.J.Suppl. 148, 213-231 (Sept. 2003).

[3] Kamionkowski, M., Kosowsky, A., and Stebbins, A., "A probe of primordial gravity waves and vorticity," Phys. Rev. Lett. 78, 2058-2061 (Mar 1997).

[4] Seljak, U. and Zaldarriaga, M., "Signature of gravity waves in polarization of the microwave background," Phys.Rev.Lett. 78, 2054-2057 (1997).

[5] Sheehy, C. et al., "The Keck Array: a pulse tube cooled CMB polarimeter," in [Society of Photo-Optical Instrumentation Engineers (SPIE) Conference Series], Society of Photo-Optical Instrumentation Engineers (SPIE) Conference Series $\mathbf{7 7 4 1}$ (July 2010).

[6] Chiang, H. C. et al., "Measurement of Cosmic Microwave Background Polarization Power Spectra from Two Years of BICEP Data," Astrophys.J. 711, 1123-1140 (Mar. 2010).

[7] Takahashi, Y. D. et al., "Characterization of the BICEP Telescope for High-precision Cosmic Microwave Background Polarimetry," Astrophys.J. 711, 1141-1156 (Mar. 2010).

[8] Ogburn, R. et al., "BICEP2 and Keck Array operational overview and status of observations," These Proceedings (2012).

[9] Kernasovskiy, S. et al., "Optimization and sensitivity of The Keck Array," These Proceedings (2012).

[10] O'Brient, R. et al., "Antenna coupled TES bolometers for BICEP2, The Keck Array, SPIDER, and Polar," These Proceedings (2012).

[11] Kaufman, J. P. et al., "Thermal stability of the BICEP2 telescope," These Proceedings (2012). 
[12] Aikin, R. W. et al., "Optical performance of the BICEP2 telescope at the South Pole," in [Society of PhotoOptical Instrumentation Engineers (SPIE) Conference Series], Society of Photo-Optical Instrumentation Engineers (SPIE) Conference Series $\mathbf{7 7 4 1}$ (July 2010).

[13] Aikin, R. et al., "Removing Instrumental Systematics Contamination from CMB Polarimetry Data," In Preparation (2012). 